OPEN ACCESS

Edited by:

Jiehui Jiang,

Shanghai University, China

Reviewed by:

Bin Wang,

Taiyuan University of Technology,

China

Yin Guimei,

Taiyuan Normal University, China

${ }^{*}$ Correspondence:

Liqun Kuang

kuang@nuc.edu.cn

Specialty section:

This article was submitted to Neurocognitive Aging and Behavior, a section of the journal

Frontiers in Aging Neuroscience

Received: 02 October 2021

Accepted: 10 January 2022

Published: 09 February 2022

Citation:

Xing J, Jia J, Wu X and Kuang L

(2022) A Spatiotemporal Brain

Network Analysis of Alzheimer's

Disease Based on Persistent

Homology.

Front. Aging Neurosci. 14:788571.

doi: 10.3389/fnagi.2022.788571

\section{A Spatiotemporal Brain Network Analysis of Alzheimer's Disease Based on Persistent Homology}

\author{
Jiacheng Xing ${ }^{1,2}$, Jiaying Jia ${ }^{1}$, Xin $W u^{2}$ and Liqun Kuang ${ }^{1 *}$ \\ ${ }^{1}$ School of Data Science and Technology, North University of China, Taiyuan, China, ${ }^{2}$ Department of Computer Science, \\ University of Birmingham, Birmingham, United Kingdom
}

Current brain network studies based on persistent homology mainly focus on the spatial evolution over multiple spatial scales, and there is little research on the evolution of a spatiotemporal brain network of Alzheimer's disease (AD). This paper proposed a persistent homology-based method by combining multiple temporal windows and spatial scales to study the spatiotemporal evolution of brain functional networks. Specifically, a time-sliding window method was performed to establish a spatiotemporal network, and the persistent homology-based features of such a network were obtained. We evaluated our proposed method using the resting-state functional MRI (rs-fMRI) data set from Alzheimer's Disease Neuroimaging Initiative (ADNI) with 31 patients with $\mathrm{AD}$ and 37 normal controls (NCs). In the statistical analysis experiment, most network properties showed a better statistical power in spatiotemporal networks than in spatial networks. Moreover, compared to the standard graph theory properties in spatiotemporal networks, the persistent homology-based features detected more significant differences between the groups. In the clustering experiment, the brain networks on the sliding windows of all subjects were clustered into two highly structured connection states. Compared to the NC group, the AD group showed a longer residence time and a higher window ratio in a weak connection state, which may be because patients with $A D$ have not established a firm connection. In summary, we constructed a spatiotemporal brain network containing more detailed information, and the dynamic spatiotemporal brain network analysis method based on persistent homology provides stronger adaptability and robustness in revealing the abnormalities of the functional organization of patients with $A D$.

Keywords: Alzheimer's disease, brain network, functional magnetic resonance imaging, dynamic functional connectivity, persistent homology, sliding window

\section{INTRODUCTION}

Alzheimer's disease (AD) (Scheltens et al., 2021) is one of the classic chronic neurodegenerative diseases, which is considered as a common form of dementia. With the progress of this disease, it gradually spread to different brain regions, thus greatly affecting the patient's daily life (Patterson, 2018). Currently, the resting-state functional MRI (rs-fMRI) (Engels et al., 2017) has become an important technology to explore brain functional networks. A lot of early brain functional 
alterations related to $\mathrm{AD}$ pathophysiology have been reported using various brain network analysis methods (Hallquist and Hillary, 2018; Marquez and Yassa, 2019; Mill et al., 2020). Most of these methods assume that the functional connectivity is constant during the brain imaging process, which may ignore some key time-varying information of the brain network caused by healthy brain or nervous system diseases. Therefore, it is necessary to study dynamic functional connectivity based on temporal dynamics (Preti et al., 2017).

Currently, the main methods used in the dynamic functional connectivity analysis include sliding window analysis, clustering analysis, time-frequency coherence analysis, and dynamic graph theory analysis (Preti et al., 2017). Among them, a large number of studies (Chen et al., 2016, 2021; de Vos et al., 2018; Lei et al., 2021) have adopted the time-sliding window approach to analyze the time-varying domain of functional connectivity. In this way, an entire rs-fMRI time series is divided into multiple subseries. For each segment, a functional connectivity network is constructed to measure the short-term correlation between the brain regions. Thus, all networks constructed from all segments describe the dynamics of the short-term functional connectivity over time. Further, the volatility between specific time points or time windows is observed by some network metrics. Specifically, a dynamic graph theory-based analysis method has developed a series of network metrics (Hallquist and Hillary, 2018; Sporns, 2018) to detect the differences of brain network structures over time in rs-fMRI research. Using the method of sliding window, more differences between the groups in a dynamic brain network can be observed, which cannot be seen in a static brain network. Multiple time windows provide more temporal evolution information for the brain network analysis. However, the spatial evolution information is missing as the brain network is measured on a single fixed spatial scale.

Recently, persistent homology (Edelsbrunner and Harer, 2010) is an important research tool of topological data analysis, which focuses on exploring topological invariants with the increase of spatial scales. In the process of spatial scale change, the topological feature with a shorter duration is considered as noise, and the feature with a longer duration is used to represent an essential feature (Giusti et al., 2016). Compared with the traditional graph theory method, it can better reveal topological changes in space and avoid the problem of scale threshold selection. Some studies have successfully applied the persistent homology technology to analyze the brain network structure of AD (Kuang et al., 2019a, 2020b), epilepsy (Choi et al., 2014), autism spectrum disorder, attention-deficit hyperactivity disorder (Lee et al., 2012, 2017), etc. In our prior study on AD brain networks (Kuang et al., 2019a), we have proposed an integrated persistent feature (IPF) based on persistent homology that achieves holistic descriptions of spatial dynamics of the brain network. We have also found that the IPF is more robustness than graph theory-based metrics in our prior studies (Kuang et al., 2019a,b, 2020a,b). However, all existing studies on persistent homology only focus on the feature invariants in the process of spatial dynamics, but no literature studies have reported the influence of the change of time window on feature invariants.
In summary, there are a few available frameworks that can simultaneously quantify the brain network structure both in the time domain and in the spatial domain. As the time-sliding window approach can produce multiple dynamic time windows for studying the characteristics of time-varying connectivity, and the persistent homology method has been verified to be good at measuring the characteristics of space-varying connectivity, we propose a novel method combining spatial scale filtrations of persistent homology with temporal sliding windows. We hypothesize that such generated spatiotemporal network dynamics may improve the performance of detecting $\mathrm{AD}$-induced topological changes in the rs-fMRI analysis, and the derived network properties based on persistent homology may be used as the potential biomarkers for AD imaging.

In this paper, we develop a persistent homology-based method by combining multiple temporal windows and spatial scales to study the spatiotemporal evolution of brain functional networks. Specifically, a time-sliding window method is performed to establish a spatiotemporal network and the persistent homologybased features of such a network are obtained. In our experiments, we evaluate our proposed method using rs-fMRI data set with 31 patients with $\mathrm{AD}$ and 37 normal controls (NCs). We test whether the proposed method provides stronger adaptability and robustness in revealing the abnormalities of the functional organization of patients with $\mathrm{AD}$.

\section{MATERIALS AND METHODS}

For each participant, the processing and analysis pipeline is summarized in Figure 1. Initially, the rs-fMRI data were acquired and preprocessed using well-known toolboxes (Step 1). Then, each subject's blood oxygen-level dependent (BOLD) signals within each region of interest (ROI) were obtained and divided into some segments by moving a sliding window over it (Step 2). For each subject, we then constructed a spatial network (Step 3) from the entire BOLD signals, as well as a series of spatiotemporal networks (Step 4) from the divided signal segments. Further, we applied a clustering analysis to spatiotemporal networks to verify whether the network structures of $\mathrm{AD}$ and NC are different (Step 5). Finally, we measured the network topology properties (Step 7) of each subject using the two kinds of methods, i.e., traditional graph theory and novel persistent homology based on graph filtration (Giusti et al., 2016) (Step 6). The specific steps are described in the following subsections.

\section{Data Acquisition and Preprocessing}

The data used in the preparation of this article were obtained from the Alzheimer's Disease Neuroimaging Initiative (ADNI) database $^{1}$ (Jack et al., 2008; Jagust et al., 2010), which are used to measure the progression of mild cognitive impairment (MCI) and early AD. The rs-fMRI data set was acquired from ADNI-2 in this study. Specifically, the 3.0T Philips MRI scanner was used to scan the brain of the subjects, and a high-resolution rs-fMRI three-dimensional image was acquired by an echo plane imaging

\footnotetext{
${ }^{1}$ adni.loni.usc.edu
} 


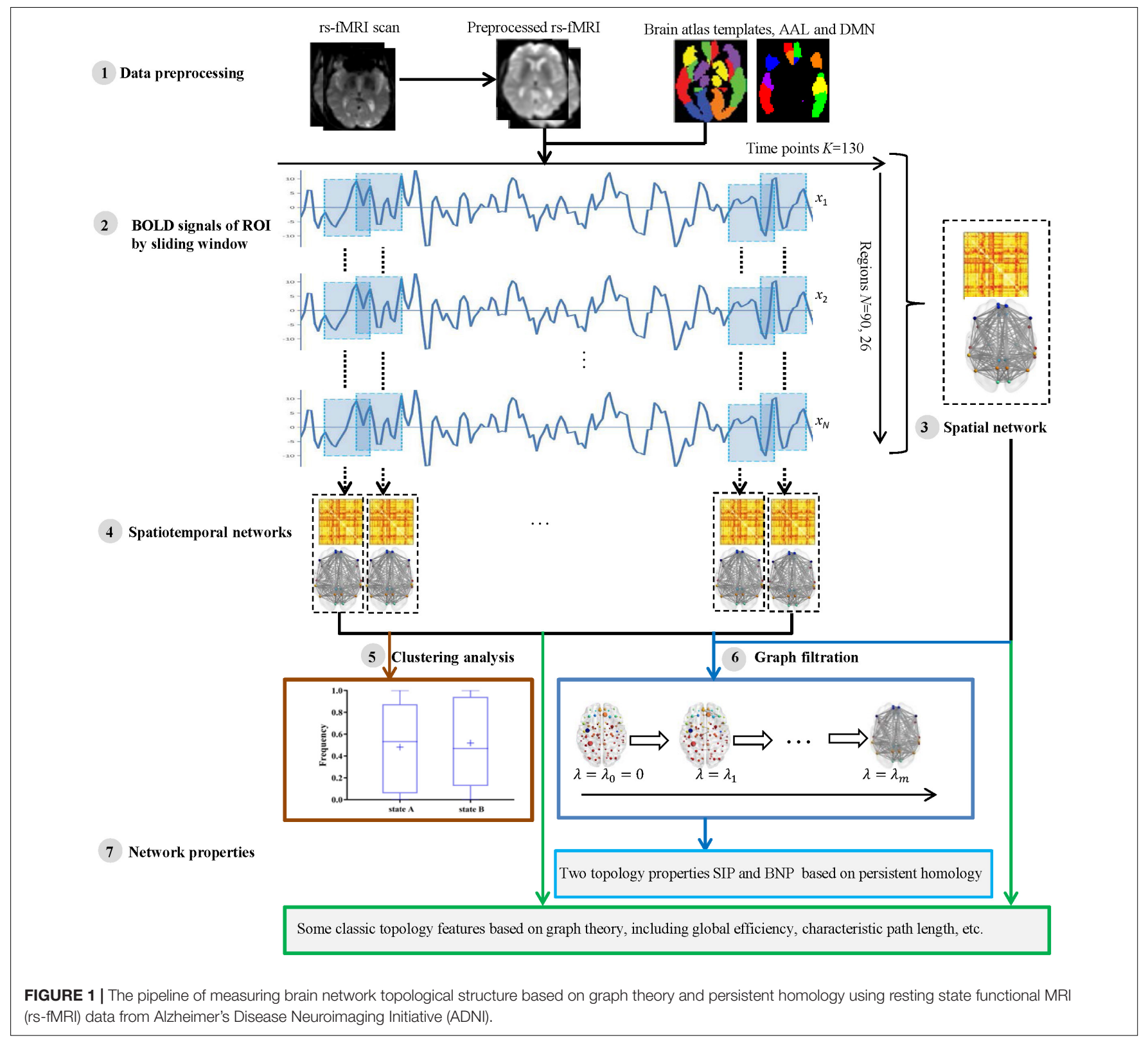

(EPI) sequence. The acquisition parameters are as follows: echo time $(\mathrm{TE})=30 \mathrm{~ms}$, turning angle $=80^{\circ}$, slice thickness $=3.3 \mathrm{~mm}$, slice number $=48$, matrix size $=64 \times 64$, repetition time $(\mathrm{TR})=3,000 \mathrm{~ms}$, and time point volume $=140$.

In this study, we used the commonly available rs-fMRI data of the ADNI database and screened the subjects' age, education level, and gender. Thus, there were a total of $68 \mathrm{AD}$ and $\mathrm{NC}$ studies. Then, spm $12^{2}$ and DPARSF toolboxes ${ }^{3}$ (Yan and Zang, 2010) were applied for rs-fMRI data preprocessing. The specific steps are as follows.

Firstly, for each subject, the first 10 time points of rs-fMRI images were discarded to improve the signal-to-noise ratio and

${ }^{2}$ http://www.fil.ion.ucl.ac.uk/spm/

${ }^{3} \mathrm{http}: / /$ restfmri.net/forum/DPARSF achieve signal stability. The remaining 130 time points were used for further analysis.

Secondly, the time correction was applied according to the acquisition time difference between the scanning layers. The slice at TR/2 time point was used as the reference scanning layer, and other scanning layers were aligned to it to ensure that the acquisition of each slice corresponds to the same time point.

Further, a spatial correction was applied. Each subject's functional images were aligned to the Montreal Institute of Neurology (MNI) space according to its structural image ( $\mathrm{T} 1$ image).

Then, spatial smoothing with a Gaussian kernel of $4 \times 4 \times 4 \mathrm{~mm}$ full width at half maximum, linear trend removal, band-pass filtering (0.01-0.08 Hz), and global signal regression were carried out in turn. 
Finally, a covariate regression analysis was used to eliminate the signal fluctuation caused by a cerebrospinal fluid signal, white matter signal, and head movement.

As there is still a controversy on whether to eliminate the whole brain signal, this study did not regress the whole brain signal. At the end of the experiment, the data of head displacement more than $1 \mathrm{~mm}$ or of head-motion rotation more than $1^{\circ}$ were excluded.

\section{Construction of Brain Networks}

We constructed the two modes of brain network, i.e., spatial network and spatiotemporal network. A spatial network was established according to the brain partition template on spatially anatomical labeling, while a spatiotemporal network was constructed by sliding a time series window.

\section{Construction of Spatial Network}

According to standard automated anatomical labeling (AAL) atlas (Tzourio-Mazoyer et al., 2002), the whole brain is spatially divided into 90 ROIs. In this paper, we also studied another subnetwork of AAL with 26 ROIs, i.e., default mode network (DMN). After image preprocessing, the average rs-fMRI time series (a total of 130 time series, $K=130$ ) of each brain region were summarized, and the Pearson correlation coefficient between each pair of ROIs was defined as its functional connectivity. Therefore, the spatial brain network of each subject was constructed and represented as an adjacency matrix with a size of $90 \times 90$ or $26 \times 26(N=90$ or 26$)$. Specifically, each ROI represents a node, and the edge weight between ROI $i$ and $j$ is defined based on Pearson correlation, i.e.,

$$
\begin{aligned}
& w_{i j}=1-\frac{\operatorname{cov}\left(x_{i}, x_{j}\right)}{\sigma_{x_{i}} \sigma_{x_{j}}}= \\
& 1-\frac{\sum_{k=1}^{N}\left(x_{i k}-\bar{x}_{i}\right)\left(x_{j k}-\bar{x}_{j}\right)}{\sqrt{\sum_{k=1}^{N}\left(x_{i k}-\bar{x}_{i}\right)^{2}} \sqrt{\sum_{k=1}^{N}\left(x_{j k}-\bar{x}_{j}\right)^{2}}},
\end{aligned}
$$

where $x_{i}=\left(x_{i 1}, x_{i 2}, \cdots, x_{i N}\right)$ and $x_{j}=\left(x_{j 1}, x_{j 2}, \cdots, x_{j N}\right)$ are the time series of BOLD signals at the $i$ th and $j$ th ROI, respectively.

\section{Construction of Spatiotemporal Network Based on Sliding Time Window}

In this study, a sliding window method (Chen et al., 2016, 2021; de Vos et al., 2018; Lei et al., 2021) was performed to establish a spatiotemporal network as shown in Figure 2. A detailed construction process is described as follows.

First, a conical window was created by convoluting a sliding time series window with a Gaussian kernel ( $\sigma=3 \mathrm{TR})$. Compared with the traditional rectangle, the conical window has the advantage of reducing the weight of boundary time points when the subsequence window is sliding. The sliding window width $W$ was set to 20TR (60 s) because previous studies have shown that a window width of 30-60 s is able to successfully capture restingstate functional connectivity fluctuations (Preti et al., 2017).

Then, for each subject, we moved the sliding window gradually in the step of one TR each time and thus produced $(K-W+1)$ subsequences of time windows. Here, $K=130$ is the length of the time series.

Further, for each time window $i$, an observation distance matrix $C_{i}(i=1,2, K-W+1)$ was generated based on Pearson correlation according to Equation (1), and the size of matrix is $N \times N(N$ is the number of ROI, $N=90$ or 26$)$.

Thus, each subject had a series of time-varying matrices, which could capture the dynamic changes of functional connectivity during the resting-state scanning. A time-varying matrix is used as a spatiotemporal network (see step 4 in Figure 1).

Finally, to quantify fluctuations in the connectivity time courses, some summary measures have been used, such as their SD (Preti et al., 2017; de Vos et al., 2018) or covariance (Chen et al., 2016). In this study, for each subject, we calculated a SD matrix across all time-varying matrices to quantitatively estimate the stability of functional connectivity over time.

\section{Spatiotemporal Network Clustering Analysis}

In this study, we propose a novel method based on persistent homology to detect the differences of dynamic functional networks between $\mathrm{AD}$ and $\mathrm{NC}$ groups. However, the mostly applied strategy in this area is the state extraction of dynamic functional connectivity through $k$-means clustering (Preti et al., 2017). To verify the effectiveness of our proposed method, we also use the traditional method to conduct experiments on spatiotemporal networks. Specifically, we used $k$-means clustering (de Vos et al., 2018) to capture the state changes of functional connectivity in a dynamic time series, and then analyzed the differences between the groups of all patients with $\mathrm{AD}$ and NC subjects in the identical states of dynamic functional connectivity. The $k$-means clustering method uses a distance function to cluster data into different clusters in an iterative manner. It clustered the time-varying matrices of all sliding windows of all individuals into $k$ clustering states. The similarity within the cluster is very high, while the similarity between the clusters is extremely low. In this study, the $k$-means algorithm was applied to a series of time-varying matrices according to its Manhattan distance (Allen et al., 2014).

We further observed each clustering state from two aspects, window ratio and average residence time using the DynamicBC toolbox (Liao et al., 2014). Specifically, the window ratio is calculated as the proportion of staying in a given state window, and the average residence time represents the number of consecutive windows belonging to a state.

\section{Network Properties}

In this study, network measurement methods based on graph theory and persistent homology were used to measure brain functional organization.

\section{Graph Theory-Based Network}

Graph theory analysis methods (Sporns, 2018) have been widely used in the study of brain network topology structure. This paper mainly used the currently widely used graph theory indicators. We measured the global and local transmission capabilities of the network (global efficiency and local efficiency) 


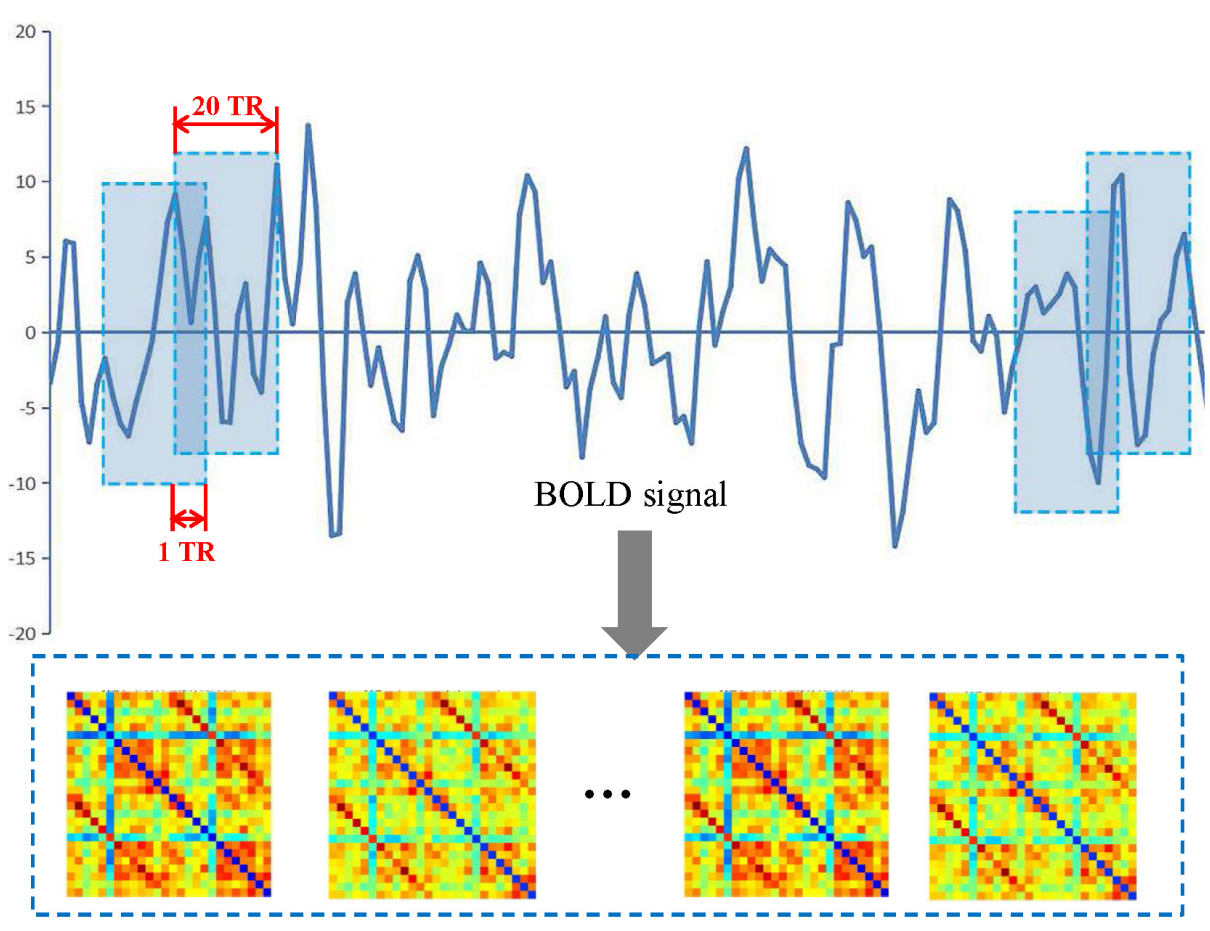

Time-varying observation distance matrices

FIGURE 2 | Construction of a spatiotemporal brain network by the sliding window method.

(De Pasquale et al., 2016), the shortest path between the brain regions (characteristic path length) (Brier et al., 2014), the importance of nodes (eigenvector centrality) (Binnewijzend et al., 2014), the degree of clustering of nodes (clustering coefficient) (Rauchmann et al., 2021), the characteristics of the smallworld network (Watts and Strogatz, 1998), and the minimum eccentricity of nodes (network radius) (Fujita et al., 2017). As these graph theory indicators usually measure the brain network on a fixed scale, the brain network needs to be filtered before measuring its structure. The statistical analysis of connectivity has been commonly used to filter the network. Specifically, we deleted the edges that were not significantly connected in the brain network $(p>0.05)$ to obtain a sparse structure (Smith and Nichols, 2009; Wang et al., 2013). Then, we applied a brain connectivity toolbox ${ }^{4}$ (Rubinov and Sporns, 2010) on the filtered brain network to calculate these graph theory-based indicators.

\section{Persistent Homology-Based Network Properties}

Persistent homology (Edelsbrunner and Harer, 2010) is a mathematical concept from the algebraic topology. The typical approach of persistent homology is Betty number plot (BNP) (Lee et al., 2012; Giusti et al., 2016), which can distinguish persistent features from noise in graph filtering and is considered as a useful feature descriptor. It has been successfully used in brain network research based on fluorodeoxyglucose-positron emission tomography (FDG-PET) and MRI data in some

${ }^{4}$ https://sites.google.com/site/bctnet/ neurodegenerative diseases (Lee et al., 2012, 2017; Choi et al., 2014). In our previous research, we proposed an IPF (Kuang et al., 2019a), which integrates a connected component aggregation cost with BNP to realize the spatial evolution of the overall graph. The IPF is defined as follows:

$$
I P F_{\lambda_{i}}=\left\{\begin{array}{cc}
\frac{m-i}{m(m-1)} \sum_{k=i+1}^{m-1} \lambda_{k}, & 0 \leq i \leq m-2 \\
0 . & i=m-1
\end{array},\right.
$$

where $m$ is the maximum number of connected components, $\lambda$ is a series of filtration values $\left(\lambda_{0}=0<\lambda_{1}<\lambda_{2}<\ldots<\lambda_{m-1}\right)$ generated by all edge weights of the maximum connected components of the brain network (see step 6 in Figure 1).

Further, the medical field tends to use a single indicator to measure biomarkers. Because IPF is a monotonically decreasing convergence function, the slope of IPF (SIP) is used as an index to quantify the dynamic research of AD brain network. We have successfully applied the SIP to AD brain network analysis in our prior studies (Kuang et al., 2019a,b, 2020a,b). Our opensource code of persistent homology can be downloaded at http://gsl.lab. asu.edu/software/IPF.

\section{Statistical Test}

A two-sample $t$-test was performed on the brain network properties based on graph theory and persistent homology, as well as clustering states. $p<0.05$ indicates that the difference 
between the two groups is statistically significant. We used Matlab (R2017a) for statistical test.

\section{RESULTS}

\section{Demographic Information}

The experimental participants were obtained from ADNI-2. ${ }^{5}$ Details of demographic characteristics are shown in Table $\mathbf{1 .}$ There were 31 AD subjects aged 60-90 years old in rsfMRI data set. To conduct a comparative study with the patient group, 37 matched NC subjects were selected in this study. Diagnostic classification was made by ADNI investigators using the established criteria. The Clinical Dementia Rating (CDR) global score of any $\mathrm{AD}$ patient was no less than 1, while that of NC subject was 0 . There are no significant differences in age, education, and gender between the groups. However, there is a significant difference in Mini-Mental Status Examination (MMSE) scores (Folstein et al., 1975). In addition, the displacement of head movement in any subject's image is less than $1 \mathrm{~mm}$, and the rotation angle in any direction is less than $1^{\circ}$.

\section{Whole Brain Network Properties}

In this study, the properties based on graph theory and persistent homology methods were used for the whole brain network analysis using AAL atlas. Their statistical differences between groups are shown in Table 2 using a two-sample $t$-test.

In the network measurement using graph theory-based properties, the network radius showed significant differences between patients with $\mathrm{AD}$ and subjects with $\mathrm{NC}(A D: 6.17 \pm 2.63$; $N C: 5.97 \pm 2.64, p=0.0493)$ in a spatial network. However, other network properties based on the graph theory could not detect any significant differences between the groups either in a spatial or spatiotemporal network.

Then, in the network measurement using persistent homology-based properties, the SIP index showed very significant differences between the groups in a spatial network $(A D:-0.67 \pm 0.13 ; N C:-0.75 \pm 0.10, p=0.008<0.01)$, so does the BNP index $(A D:-257.16 \pm 43.18$; NC: $-283.12 \pm 56.82$, $p=0.041<0.05)$. Moreover, in the spatiotemporal network measurement, the group difference of BNP is more significant

${ }^{5}$ http://adni.loni.usc.edu

TABLE 1 | Demographic information of experimental subjects.

\begin{tabular}{lccc}
\hline & AD $(\boldsymbol{n}=\mathbf{3 1})$ & $\mathbf{N C}(\boldsymbol{N}=\mathbf{3 7})$ & $\boldsymbol{p}$-value \\
\hline Age & $74.0 \pm 6.1$ & $74.1 \pm 6.2$ & 0.7486 \\
Education & $15.4 \pm 3.9$ & $16.1 \pm 3.6$ & 0.4267 \\
Gender (male/female) & $16 / 15$ & $15 / 22$ & 0.6033 \\
MMSE & $22.8 \pm 3.4$ & $28.8 \pm 1.6$ & 0.0015 \\
CDR score & $\geq 1$ & 0 & -
\end{tabular}

Data are presented as mean $\pm S D$.

$A D$, Alzheimer's disease; NC, normal control; MMSE, Mini-Mental State Examination; $C D R$, clinical dementia rating.
$(A D:-718.53 \pm 84.92 ; N C:-793.13 \pm 109.96, p=0.0030$, much less than 0.041) than in a spatial network, and so is SIP $(A D:-0.52 \pm 0.11 ; N C:-0.59 \pm 0.09, p=0.002<0.008)$.

To sum up, the differences of persistent homology indicators between $\mathrm{AD}$ and $\mathrm{NC}$ are significantly greater than those of graph theory indicators. Moreover, their group differences in a spatiotemporal network are more significant than those in a spatial network. It suggests that a brain spatiotemporal network analysis based on persistent homology is more likely to explore potential biomarkers.

\section{Brain Default Mode Network Properties}

Default mode network is a functional subnetwork with the strongest variability in the study of $\mathrm{AD}$, which contains the most obvious areas of brain atrophy. In this study, 26 areas (Vriend et al., 2018) in AAL were identified as the ROIs of DMN. The statistical differences of network properties between the groups in the DMN atlas are shown in Table 3.

In the study of DMN based on the graph theory method, there is no significant difference in the small-world attribute in the spatial DMN ( $p>0.05)$, but it shows a very significant difference in the spatiotemporal network $(A D: 1.03 \pm 0.02 ; N C$ : $1.02 \pm 0.01, p=0.007)$, suggesting a small-world attribute could detect subtle time variability in the spatiotemporal DMN. In addition, it can be seen that the eigenvector centrality has very significant differences between the groups in both the spatial network ( $A D: 0.1046 \pm 0.01 ; N C: 0.1047 \pm 0.01, p=0.009)$ and spatiotemporal network $(A D: 0.1946 \pm 0.01 ; N C: 0.1950 \pm 0.01$, $p=0.017$ ), while there is no difference in the whole brain study.

Then, in the study of DMN based on persistent homology, both SIP and BNP detected significant differences between the groups in spatial and spatiotemporal networks. Especially in the spatiotemporal DMN, the SIP $(A D:-0.69 \pm 0.12$; $N C:-1.46 \pm 0.10, p=0.003)$ and BNP $(A D:-171.41 \pm 32.24$; $N C:-198.84 \pm 34.08, p=0.001)$ have very significant group differences.

In general, the spatiotemporal DMN properties based on persistent homology performed the best. Research on the persistent homology of spatiotemporal DMN is more likely to distinguish $\mathrm{AD}$ from $\mathrm{NC}$, and it is more likely to explore the potential biomarkers for $\mathrm{AD}$ imaging.

\section{Spatiotemporal Network Clustering Results}

To determine the optimal number of clusters $k$, we took $k$ from 2 to 9 and repeated the test 100 times for each value. The experimental result is shown in Figure 3, where the solid point represents the best number of clusters. Here, the most suitable number of clusters was $k=2$. The Silhouette score and Calinski-Harabasz index were used to evaluate the effectiveness of the clustering results. We further check the optimal number of clusters using a fivefold cross-validation. The average distance of test sets was calculated while selecting different cluster numbers, as shown in Figure 4. The error distance reached the minimum when the cluster number was 2 , suggesting that the optimal value of $k$ was 2 again. Thus, the $\mathrm{AD}$ and $\mathrm{NC}$ subjects' varying-time 
TABLE 2 | Statistical p-values of different network properties between Alzheimer's disease (AD) and normal control (NC) groups in automated anatomical labeling (AAL).

\begin{tabular}{|c|c|c|c|c|c|c|c|c|c|}
\hline \multirow[t]{2}{*}{ Network mode } & \multicolumn{6}{|c|}{ Graph theory-based properties } & \multicolumn{3}{|c|}{ Persistent homology-based properties } \\
\hline & GE & LE & CPL & EC & CC & sw & NR & SIP & BNP \\
\hline Spatial network & 0.075 & 0.828 & 0.484 & 0.092 & 0.357 & 0.763 & $0.049^{\star}$ & $0.008^{\star \star}$ & $0.041^{*}$ \\
\hline Spatiotemporal network & 0.230 & 0.642 & 0.518 & 0.086 & 0.327 & 0.119 & 0.311 & $0.002^{\star \star}$ & $0.003^{\star \star}$ \\
\hline
\end{tabular}

${ }^{*} p<0.05 ;{ }^{* *} p<0.01$.

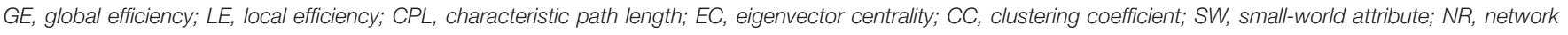
radius; SIP, slope of integrated persistent feature plot; BNP, Betty number plot.

TABLE 3 | Statistical p-values of different network properties between AD and NC groups in a default mode network (DMN).

\begin{tabular}{|c|c|c|c|c|c|c|c|c|c|}
\hline \multirow[t]{2}{*}{ Network mode } & \multicolumn{6}{|c|}{ Graph theory-based properties } & \multicolumn{3}{|c|}{ Persistent homology-based properties } \\
\hline & GE & LE & CPL & EC & CC & sw & NR & SIP & BNP \\
\hline Spatial network & 0.058 & 0.960 & 0.619 & $0.009^{\star \star}$ & 0.230 & 0.074 & 0.164 & $0.004^{\star \star}$ & $0.049^{\star}$ \\
\hline Spatiotemporal network & 0.177 & 0.447 & 0.353 & $0.017^{*}$ & 0.145 & $0.007^{\star \star}$ & 0.547 & $0.003^{\star *}$ & $0.001^{\star *}$ \\
\hline
\end{tabular}

${ }^{*} p<0.05 ;{ }^{* *} p<0.01$.

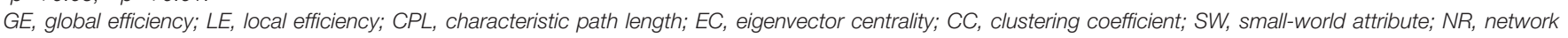
radius; SIP, slope of integrated persistent feature plot; BNP, Betty number plot.
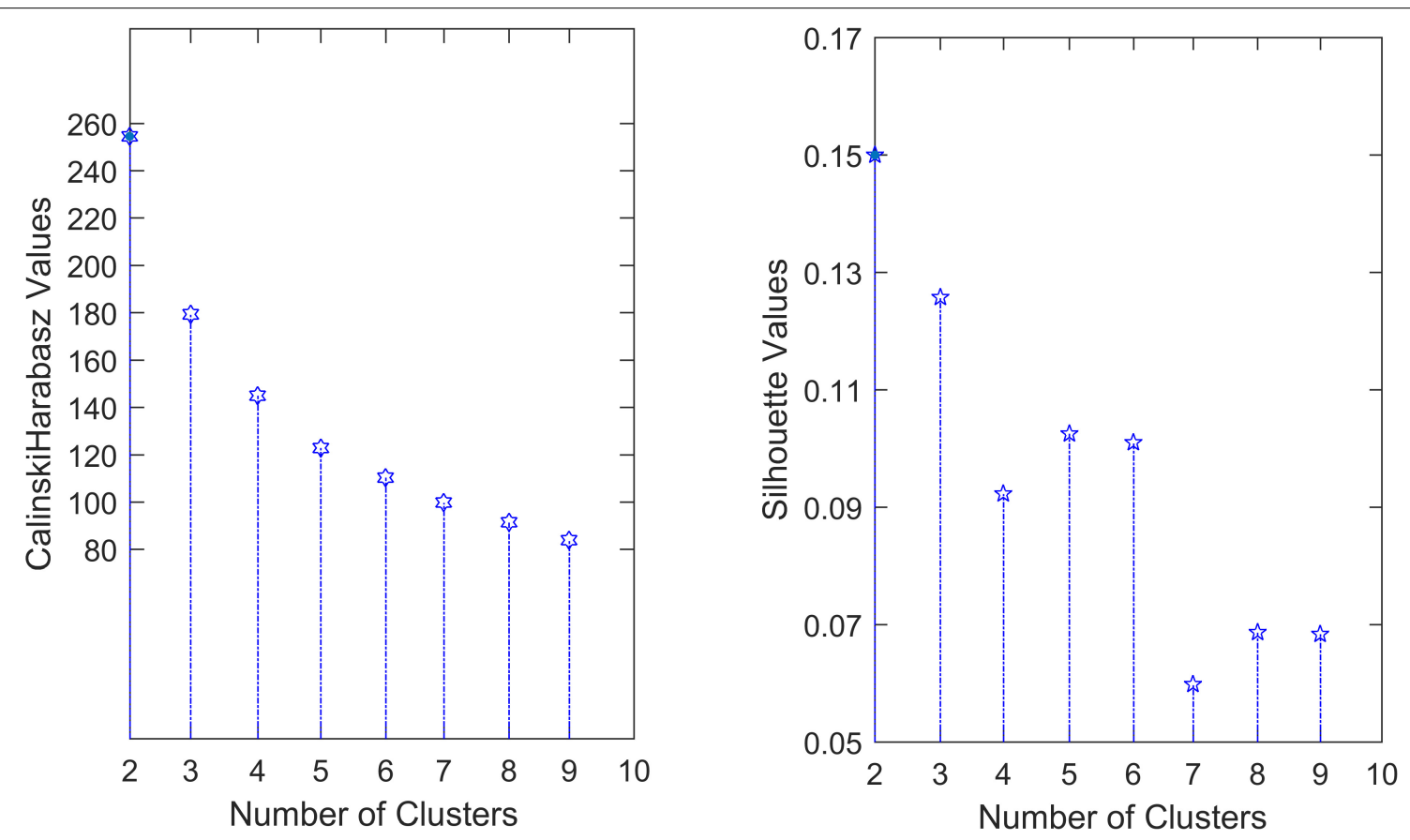

FIGURE 3 | Optimization for the number of clusters.

matrices of sliding windows were clustered into two highly structured functional connectivity states separately.

We calculated a specific functional connectivity matrix (i.e., cluster center) for all windows in each state, and each element in the matrix was the median value of the corresponding element belonging to a state in all window matrices. The cluster center diagrams of the whole brain network and DMN are shown in Figures 5, 6, respectively, where maps correspond to the number of windows in a state, and the color bar represents the Pearson correlation coefficient value. It can be seen that state A is a stronger connection state, and state B is a sparser one. In the whole brain network clustering (see Figure 5), the window ratio of patients with $\mathrm{AD}$ in state $\mathrm{A}$ is reduced by about $5 \%$ compared with NC subjects, while it increases accordingly in state B. Similarly, in the DMN clustering (see Figure 6), compared with $\mathrm{NC}$ subjects, the window ratio of patients with $\mathrm{AD}$ in state $\mathrm{B}(A D$ : $0.67 \pm 0.05 ; N C: 0.47 \pm 0.06, p<0.05)$ is significantly higher.

Further, the results of average residence time are shown in Figures 7, 8. It is calculated by averaging the number of consecutive windows before changing from one to another state. 


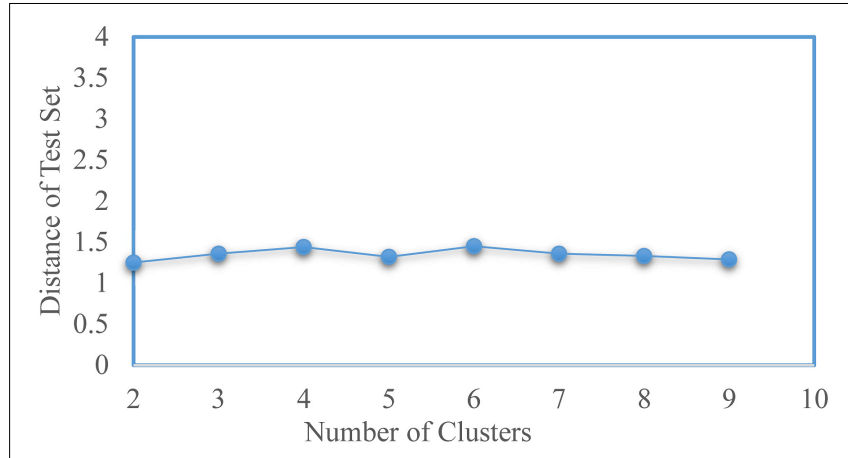

FIGURE 4 | Cross-validation to determine the number of clusters.

The number of consecutive windows of $\mathrm{AD}$ in state $\mathrm{B}$ is relatively large, either in the whole brain network (see Figure 7, AD: $40 \pm 8$ windows; NC: $27 \pm 5$ windows, $p<0.05$ ), or in DMN (see Figure 8, $A D: 47 \pm 8$; $N C: 28 \pm 6, p<0.05)$.
In summarily, the clustering results of the DMN subnetwork were similar to those of the whole brain network, and the difference between the AD and NC group was more significant. The brain network of patients with $\mathrm{AD}$ is more likely to stay in state $\mathrm{B}$, a weak connection state.

\section{DISCUSSION}

\section{Present Findings}

This paper studies the differences of spatiotemporal network dynamics between patients with $\mathrm{AD}$ and $\mathrm{NCs}$ in the whole brain network and its subnetwork DMN. There are three main findings in this study.

First, the spatiotemporal dynamics method combining a sliding window and persistent homology performs better than existing methods in the $\mathrm{AD}$ brain network analysis, and the persistent homology-based measures can be used as a potential biomarker. In our experiment, most of the network properties were more powerful in the spatiotemporal brain network than in
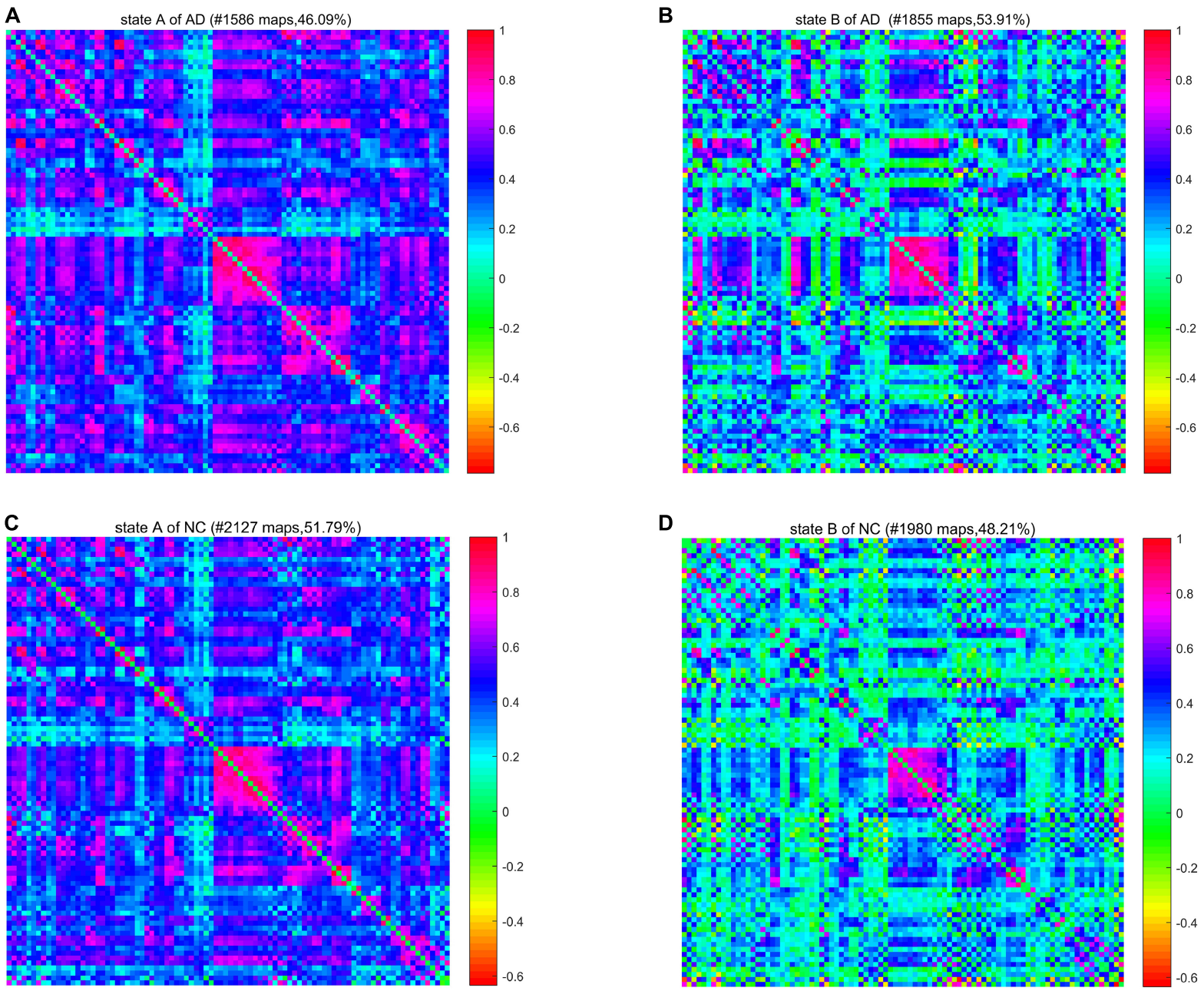

FIGURE 5 | The cluster center diagram of spatiotemporal automated anatomical labeling (AAL) networks for Alzheimer's disease (AD) and normal control (NC). 

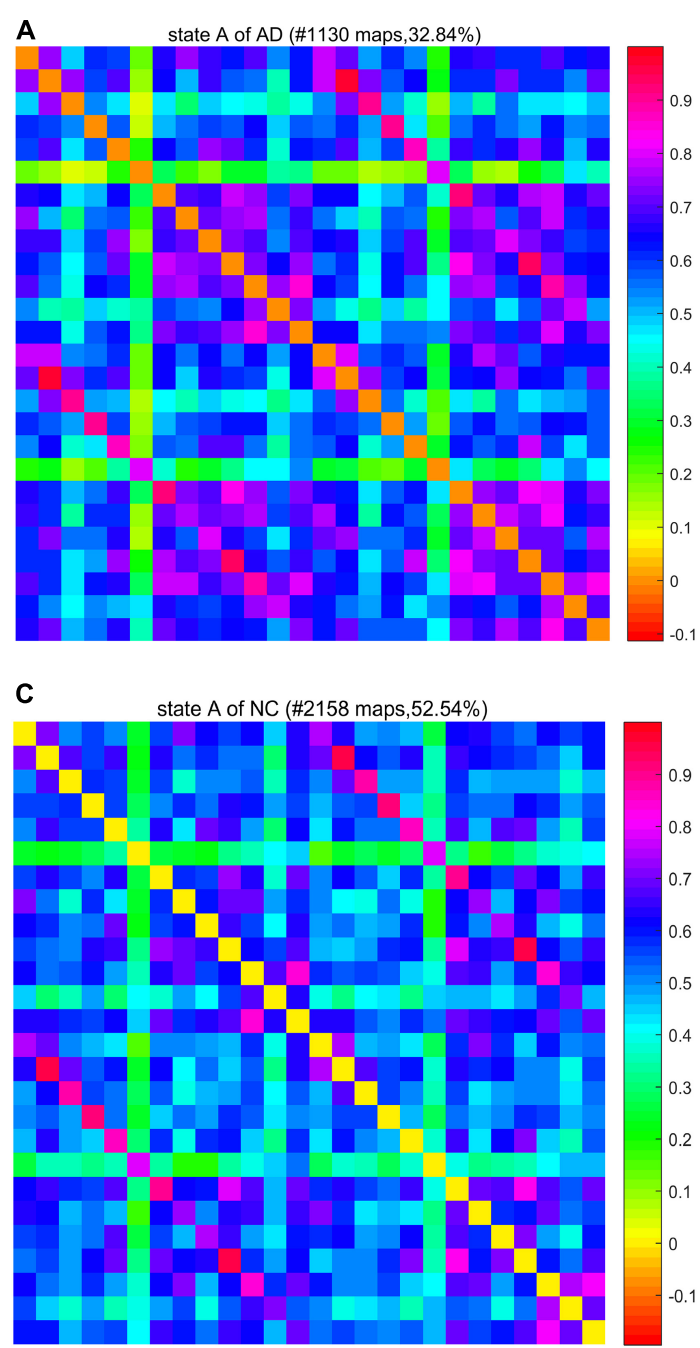

B

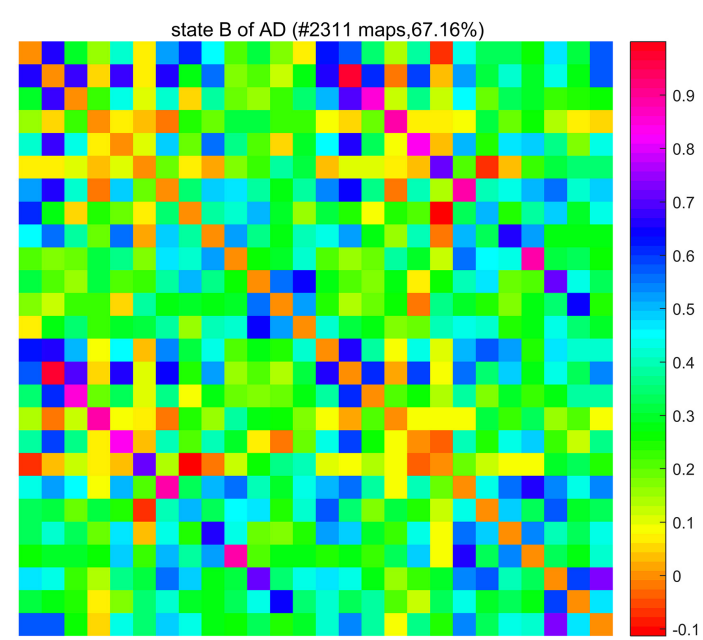

D

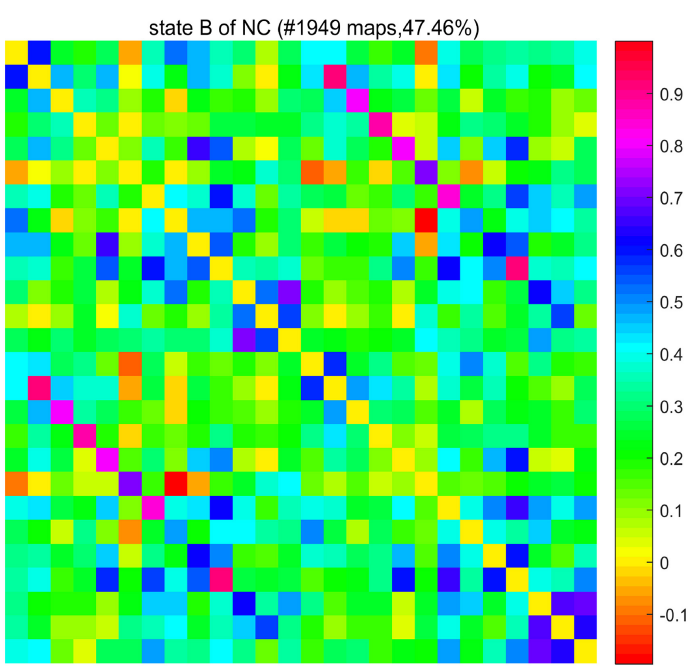

FIGURE 6 | The cluster center diagram of spatiotemporal default mode networks (DMNs) for AD and NC.

the spatial brain network, particularly two persistent homologybased properties. Both SIP and BNP detected significant group differences $(p<0.01)$ in a spatiotemporal network after introducing time-varying information, in both the whole brain network and DMN subnetwork. In addition, in the DMN, the small-world attribute of a spatiotemporal network $(p<0.01)$ is significantly different from that of a spatial network $(p>0.05)$. In general, the brain spatiotemporal network could provide a more subtle temporal variability than a spatial network.

Second, in the study on the AD brain network, the DMN is a more suitable network than the whole brain network. The difference between groups of most network properties in DMN was more apparent than that in the whole brain network. In the DMN measurement using the graph theory method, the eigenvector centrality detected very significant differences between the groups in both spatial and spatiotemporal networks, while there was no difference in the whole brain study. Similarly, there was no significant difference in the small-world attribute in the whole brain network, but there was a very significant difference in the spatiotemporal DMN. Overall, the DMN is more likely to distinguish $\mathrm{AD}$ from $\mathrm{NC}$, and it is more likely to explore the potential biomarkers for $\mathrm{AD}$ imaging.

Finally, the clustering results show that $\mathrm{AD}$ tends to be in the weak connection state. The window ratio and the average residence time in the weak connection state were relatively higher for patients with $\mathrm{AD}$ than those of NC. It may be due to the fact that patients with $\mathrm{AD}$ have not established a firm connection.

\section{Experimental Result Analysis of Proposed Method}

As the persistent homology-based features are monotonically decreasing convergence functions according to filtration values, both the network properties BNP and SIP can be considered as the information diffusion rate or convergence rate in the process of reaching a fully connected component. In this study, in both the whole brain network and DMN, the values of the BNP group and the SIP group showed the same pattern of 


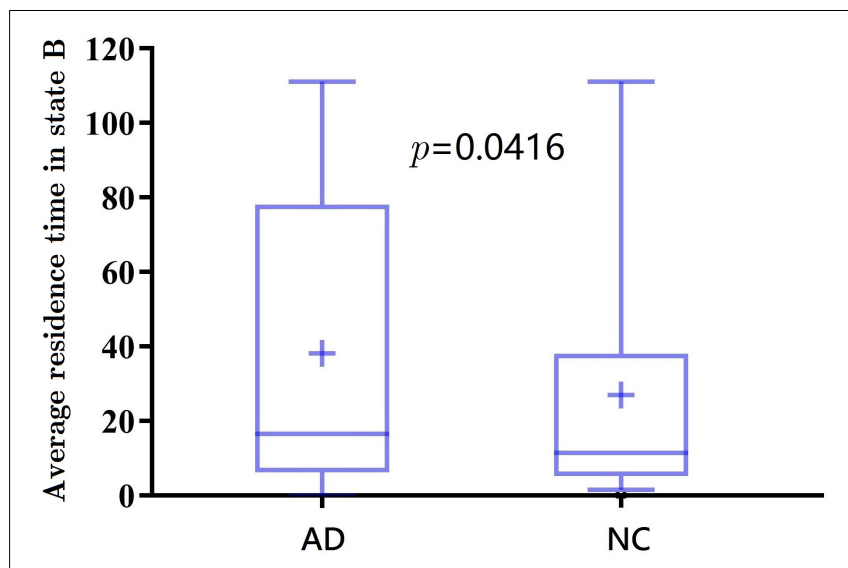

FIGURE 7 | The average residence time of spatiotemporal AAL networks for $A D$ and NC.

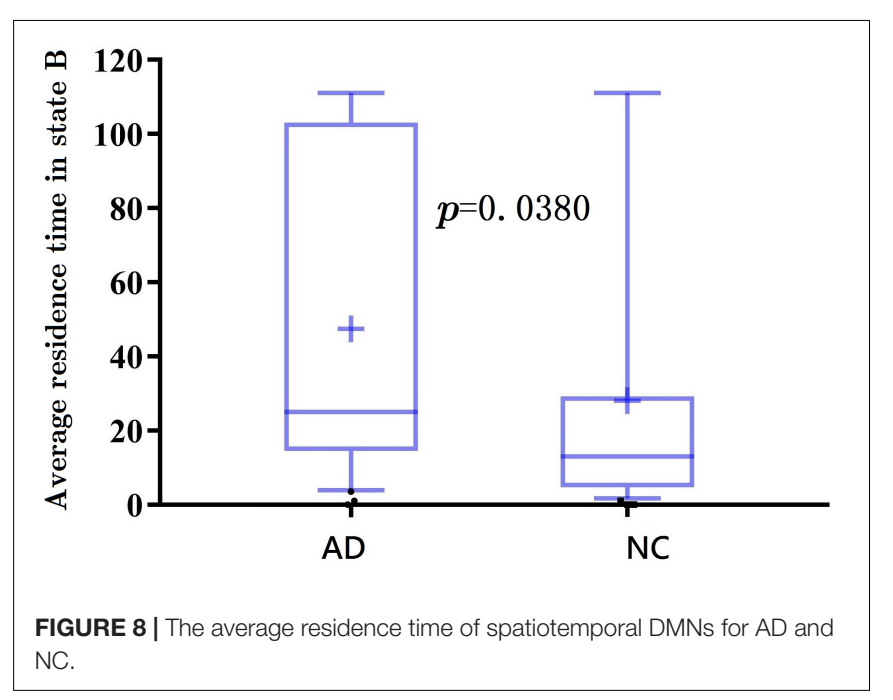

$|\mathrm{AD}|<|\mathrm{NC}|$, with a significance in the level $p<0.01$ based on a two-sample $t$-test. The fact that the BNP and SIP curves of the $\mathrm{AD}$ group are not as steep as those of the NC group may mean that the information diffusion speed in the $\mathrm{AD}$ group is slow. Because the persistent homology-based features measure the topology of the whole brain network, it is reasonable to speculate that the slow convergence rate is caused by the reduction of functional integration of the whole brain, which may further lead to cognitive defects in patients with $\mathrm{AD}$.

Further, we apply the traditional method of cluster analysis to verify the abovementioned assumption. The spatiotemporal networks of $\mathrm{AD}$ and $\mathrm{NC}$ individuals were clustered into two states. One shows the pattern with a stronger connectivity in the brain region, and another presents the pattern with less connection definitions and a lower connectivity. Moreover, the brain network of patients with $\mathrm{AD}$ were more likely to stay in a weak connection state. This finding confirms the abovementioned assumption about the weak integration ability of AD brain network. Therefore, our proposed method based on persistent homology provides a novel viewpoint of the overall tissue injury and the interruption of neuronal integration in patients with $\mathrm{AD}$ dementia.

\section{Clinical Potential of Spatiotemporal Brain Network}

We proposed a novel method to construct and measure a spatiotemporal network based on persistent homology (i.e., spatiotemporal network + persistent homology-based properties). We compared our experimental results with two dynamic spatiotemporal methods and two static spatial methods for a functional connectivity analysis, including spatiotemporal network + graph theory-based properties, spatiotemporal network $+k$-means clustering, spatial network + persistent homology-based properties, and spatial network + graph theorybased properties. Because the strength of functional connectivity varies in the range of seconds to minutes, it is important to analyze the differences between different time periods, forming temporal dynamics. Only a spatiotemporal brain network is able to capture the inner dynamic nature of connectivity alterations, therefore the dynamic spatiotemporal methods are demonstrated to be more powerful to distinguish the $\mathrm{AD}$ group from the NC group than the static spatial methods without time-varying information, as shown in Tables 2, 3.

Further, in dynamic spatiotemporal network analyses, after sliding time windows, the state extraction through $k$-means clustering and the network topology measurement have been the two most widely applied strategies (Preti et al., 2017). The former technique enables to detect differences between $\mathrm{AD}$ and NC groups based on the dynamical occurrence and connectivity strength of connectivity states. Then, the latter technique allows to refine the meaning of the observed spatial differences across groups using some network metrics. With graph theory-based network properties, various non-trivial topological features, including small-world organization, modular structure, and highly connected hubs, have been observed to be disrupted in patients with AD (Sporns, 2018; Kuang et al., 2019a). Moreover, the persistent homology-based network properties allow to address dynamic abnormalities at a more global level, where the evolution of the global pattern of connectivity contributed by all scales is probed.

In summary, the dynamic spatiotemporal network analysis provides more completed functional alterations in connectivity, which cannot be depicted by a stationary analysis. Such kinds of technique have a greater potential for clinical applications in AD.

\section{Limitation and Future Works}

Although the promising results were obtained by applying the two suggested network properties SIP and BNP based on persistent homology to discriminate spatiotemporal networks between $\mathrm{AD}$ and $\mathrm{NC}$ groups, there are some important caveats. First, most network properties showed a better statistical power in spatiotemporal networks in ADNI data set. To validate the robustness of the proposed method, we will further study on other independent data sets. Then, current research is limited to the analysis of the rs-fMRI data collected in a single time period and does not verify multiple time periods. In the future, 
we will study the longitudinal trajectories (Dautricourt et al., 2021) of functional brain dynamics over multiple time periods. Last but not least, although this article interprets the temporal dynamics of the functional brain network well and has obtained good experimental results, a correlation analysis with behavioral data should be performed in the future.

\section{CONCLUSION}

This work measured the functional brain network structure of the whole brain network and its subnetwork DMN on rsfMRI data set. A novel method is proposed based on our prior work of persistent homology. We combine multiple temporal windows and spatial scales to study the spatiotemporal brain dynamics. Most network properties show a better statistical power in spatiotemporal networks than in spatial networks, and the persistent homology-based features detected more significant differences between AD and NC groups than the standard graph theory properties. Moreover, the brain network of patients with $\mathrm{AD}$ is more likely to stay in a weak connection state in a clustering study. To the best of our knowledge, this is the first study applying persistent homology to analyze the spatiotemporal brain network. This study offers a novel insight into revealing the abnormalities of the functional organization of patients with AD.

\section{DATA AVAILABILITY STATEMENT}

Publicly available datasets were analyzed in this study. This data can be found here: http://adni.loni.usc.edu.

\section{ETHICS STATEMENT}

Written informed consent was obtained from the individual(s) for the publication of any potentially identifiable images or data included in this article.

\section{AUTHOR CONTRIBUTIONS}

JX and LK designed the study. LK acquired the data. JX, $\mathrm{XW}$, and LK analyzed and interpreted the results of the data.

\section{REFERENCES}

Allen, E. A., Damaraju, E., Plis, S. M., Erhardt, E. B., Eichele, T., and Calhoun, V. D. (2014). Tracking whole-brain connectivity dynamics in the resting state. Cereb. Cortex 24, 663-676.

Binnewijzend, M. A. A., Adriaanse, S. M., Van der Flier, W. M., Teunissen, C. E., de Munck, J. C., Stam, C. J., et al. (2014). Brain network alterations in Alzheimer's disease measured by Eigenvector centrality in fMRI are related to cognition and CSF biomarkers. Hum. Brain Mapp. 35, 2383-2393. doi: 10.1002/hbm.22335

Brier, M. R., Thomas, J. B., Fagan, A. M., Hassenstab, J., Holtzman, D. M., Benzinger, T. L., et al. (2014). Functional connectivity and graph theory in preclinical Alzheimer's disease. Neurobiol. Aging 35, 757-768.

Chen, Q., Lu, J. M., Zhang, X., Sun, Y., Chen, W. Q., Li, X., et al. (2021). Alterations in dynamic functional connectivity in individuals with subjective cognitive decline. Front. Aging Neurosci. 13:646017. doi: 10.3389/fnagi.2021.646017
JX and JJ drafted the manuscript. JX and LK revised the manuscript. All authors contributed to the article and approved the submitted version.

\section{FUNDING}

This research was funded by the Natural Science Foundation of Shanxi Province of China (201901D111150 to LK) and the Research Project Supported by Shanxi Scholarship Council of China (2020-113 to LK).

\section{ACKNOWLEDGMENTS}

Data collection and sharing for this project were funded by the ADNI (National Institutes of Health Grant U01 AG024904) and DOD ADNI (Department of Defense award number W81XWH12-2-0012). ADNI was funded by the National Institute on Aging, the National Institute of Biomedical Imaging and Bioengineering, and through generous contributions from the following: AbbVie, Alzheimer's Association; Alzheimer's Drug Discovery Foundation; Araclon Biotech; BioClinica, Inc.; Biogen; BristolMyers Squibb Company; CereSpir, Inc.; Cogstate; Eisai Inc.; Elan Pharmaceuticals, Inc.; Eli Lilly and Company; EuroImmun; F. Hoffmann-La Roche Ltd. and its affiliated company Genentech, Inc.; Fujirebio; GE Healthcare; IXICO Ltd.; Janssen Alzheimer Immunotherapy Research \& Development, LLC.; Johnson \& Johnson Pharmaceutical Research \& Development LLC.; Lumosity; Lundbeck; Merck \& Co., Inc.; Meso Scale Diagnostics, LLC.; NeuroRx Research; Neurotrack Technologies; Novartis Pharmaceuticals Corporation; Pfizer Inc.; Piramal Imaging; Servier; Takeda Pharmaceutical Company; and Transition Therapeutics. The Canadian Institutes of Health Research is providing funds to support ADNI clinical sites in Canada. Private sector contributions are facilitated by the Foundation for the National Institutes of Health (www.fnih.org). The grantee organization is the Northern California Institute for Research and Education, and the study was coordinated by the Alzheimer's Therapeutic Research Institute at the University of Southern California. ADNI data are disseminated by the Laboratory for Neuro Imaging at the University of Southern California.

Chen, X. B., Zhang, H., Gao, Y., Wee, C. Y., Li, G., Shen, D. G., et al. (2016). HighOrder resting-state functional connectivity network for MCI classification. Hum. Brain Mapp. 37, 3282-3296. doi: 10.1002/hbm.23240

Choi, H., Kim, Y. K., Kang, H., Lee, H., Im, H.-J., Kim, E. E., et al. (2014). Abnormal metabolic connectivity in the pilocarpine-induced epilepsy rat model: a multiscale network analysis based on persistent homology. NeuroImage 99, 226-236.

Dautricourt, S., de Flores, R., Landeau, B., Poisnel, G., Vanhoutte, M., Delcroix, N., et al. (2021). Longitudinal changes in hippocampal network connectivity in Alzheimer's disease. Ann. Neurol. 90, 391-406. doi: 10.1002/ana.26168

De Pasquale, F., Della Penna, S., Sporns, O., Romani, G., and Corbetta, M. (2016). A dynamic core network and global efficiency in the resting human brain. Cereb. Cortex 26, 4015-4033.

de Vos, F., Koini, M., Schouten, T. M., Seiler, S., van der Grond, J., Lechner, A., et al. (2018). A comprehensive analysis of resting state fMRI measures to 
classify individual patients with Alzheimer's disease. Neuroimage 167, 62-72. doi: 10.1016/j.neuroimage.2017.11.025

Edelsbrunner, H., and Harer, J. (2010). Computational Topology: an Introduction. Providence, RI: American Mathematical society.

Engels, M. M. A., van der Flier, W. M., Stam, C. J., Hillebrand, A., Scheltens, P., and van Straaten, E. C. W. (2017). Alzheimer's disease: the state of the art in resting-state magnetoencephalography. Clin. Neurophysiol. 128, 1426-1437. doi: 10.1016/j.clinph.2017.05.012

Folstein, M. F., Folstein, S. E., and McHugh, P. R. (1975). "Mini-mental state": a practical method for grading the cognitive state of patients for the clinician. J. Psychiatric Res. 12, 189-198.

Fujita, A., Takahashi, D. Y., Balardin, J. B., Vidal, M. C., and Sato, J. R. (2017). Correlation between graphs with an application to brain network analysis. Comput. Stat. Data Anal. 109, 76-92. doi: 10.1016/j.csda.2016.11.016

Giusti, C., Ghrist, R., and Bassett, D. S. (2016). Two's company, three (or more) is a simplex : algebraic-topological tools for understanding higher-order structure in neural data. J. Comput. Neurosci. 41, 1-14. doi: 10.1007/s10827-016-0608-6

Hallquist, M. N., and Hillary, F. G. (2018). Graph theory approaches to functional network organization in brain disorders: a critique for a brave new small-world. Network Neurosci. 3, 1-26. doi: 10.1162/netn_a_00054

Jack, C. R., Bernstein, M. A., Fox, N. C., Thompson, P., Alexander, G., Harvey, D., et al. (2008). The Alzheimer's disease neuroimaging initiative (ADNI): MRI methods. J. Magn. Reson. Imaging 27, 685-691.

Jagust, W. J., Bandy, D., Chen, K., Foster, N. L., Landau, S. M., Mathis, C. A., et al. (2010). The Alzheimer's disease neuroimaging initiative positron emission tomography core. Alzheimer's Dementia 6, 221-229.

Kuang, L., Han, X., and Chen, K. (2019a). A concise and persistent feature to study brain resting-state network dynamics: findings from the Alzheimer's disease Neuroimaging Initiative. Hum. Brain Mapp. 40, 1062-1081. doi: 10.1002/hbm. 24383

Kuang, L., Zhao, D., Xing, J., Chen, Z., Xiong, F., and Han, X. (2019b). Metabolic Brain Network Analysis of FDG-PET in Alzheimer's disease using kernel-based persistent features. Molecules 24:2301. doi: 10.3390/molecules24122301

Kuang, L. Q., Gao, Y., Chen, Z. Y., Xing, J. C., Xiong, F. G., and Han, X. (2020a). White matter brain network research in Alzheimer's disease using persistent features. Molecules 25:2472. doi: 10.3390/molecules25112472

Kuang, L. Q., Jia, J. Y., Zhao, D. Y., Xiong, F. G., Han, X., and Wang, Y. L. (2020b). Default mode network analysis of APOE genotype in cognitively unimpaired subjects based on persistent homology. Front. Aging Neurosci. 12:188. doi: 10. 3389/fnagi.2020.00188

Lee, H., Kang, H., Chung, M. K., Kim, B.-N., and Lee, D. S. (2012). Persistent brain network homology from the perspective of dendrogram. IEEE Trans. Med. Imaging 31, 2267-2277.

Lee, H., Kang, H., Chung, M. K., Lim, S., Kim, B. N., and Lee, D. S. (2017). Integrated multimodal network approach to PET and MRI based on multidimensional persistent homology. Hum. Brain Mapp. 38, 1387-1402.

Lei, B. Y., Yu, S. Z., Zhao, X., Frangi, A. F., Tan, E. L., Elazab, A., et al. (2021). Diagnosis of early Alzheimer's disease based on dynamic high order networks. Brain Imaging Behav. 15, 276-287. doi: 10.1007/s11682-019-00255-9

Liao, W., Wu, G. R., Qiang, X., Ji, G. J., Zhang, Z., Zang, Y. F., et al. (2014). DynamicBC: a MATLAB toolbox for dynamic brain connectome analysis. Brain Connect. 4:780.

Marquez, F., and Yassa, M. A. (2019). Neuroimaging biomarkers for Alzheimer's disease. Mol. Neurodegener. 14:21. doi: 10.1186/s13024-019-0325-5

Mill, R. D., Gordon, B. A., Balota, D. A., and Cole, M. W. (2020). Predicting dysfunctional age-related task activations from resting-state network alterations. Neuroimage 221:117167. doi: 10.1016/j.neuroimage.2020.11 7167

Patterson, C. (2018). World Alzheimer Report 2018. London: Alzheimer's Disease International.

Preti, M. G., Bolton, T. A. W., and Van De Ville, D. (2017). The dynamic functional connectome: State-of-the-art and perspectives. Neuroimage 160, 41-54. doi: 10.1016/j.neuroimage.2016.12.061

Rauchmann, B.-S., Ersoezlue, E., Stoecklein, S., Keeser, D., Brosseron, F., Buerger, K., et al. (2021). Resting-State network alterations differ between Alzheimer's disease atrophy subtypes. Cereb. Cortex 31, 4901-4915. doi: 10.1093/cercor/ bhab130

Rubinov, M., and Sporns, O. (2010). Complex network measures of brain connectivity: uses and interpretations. NeuroImage 52, 1059-1069.

Scheltens, P., De Strooper, B., Kivipelto, M., Holstege, H., Chetelat, G., Teunissen, C. E., et al. (2021). Alzheimer's disease. Lancet 397, 1577-1590. doi: 10.1016/ s0140-6736(20)32205-4

Smith, S. M., and Nichols, T. E. (2009). Threshold-free cluster enhancement: addressing problems of smoothing, threshold dependence and localisation in cluster inference. Neuroimage 44, 83-98. doi: 10.1016/j.neuroimage.2008.03. 061

Sporns, O. (2018). Graph theory methods: applications in brain networks. Dial. Clin. Neurosci. 20, 111-121.

Tzourio-Mazoyer, N., Landeau, B., Papathanassiou, D., Crivello, F., Etard, O., Delcroix, N., et al. (2002). Automated anatomical labeling of activations in SPM using a macroscopic anatomical parcellation of the MNI MRI single-subject brain. NeuroImage 15, 273-289.

Vriend, C., van den Heuvel, O. A., Berendse, H. W., van der Werf, Y. D., and Douw, L. (2018). Global and subnetwork changes of the structural connectome in de novo Parkinson's disease. Neuroscience 386, 295-308. doi: 10.1016/j. neuroscience.2018.06.050

Wang, J., Zuo, X., Dai, Z., Xia, M., Zhao, Z., Zhao, X., et al. (2013). Disrupted functional brain connectome in individuals at risk for Alzheimer's disease. Biol. Psychiatry 73, 472-481. doi: 10.1016/j.biopsych.2012. 03.026

Watts, D. J., and Strogatz, S. H. (1998). Collective dynamics of 'small-world' networks. Nature 393:440.

Yan, C., and Zang, Y. (2010). DPARSF: a MATLAB toolbox for" pipeline" data analysis of resting-state fMRI. Front. Sys. Neurosci. 4:13. doi: 10.3389/fnsys. 2010.00013

Conflict of Interest: The authors declare that the research was conducted in the absence of any commercial or financial relationships that could be construed as a potential conflict of interest.

Publisher's Note: All claims expressed in this article are solely those of the authors and do not necessarily represent those of their affiliated organizations, or those of the publisher, the editors and the reviewers. Any product that may be evaluated in this article, or claim that may be made by its manufacturer, is not guaranteed or endorsed by the publisher.

Copyright (c) 2022 Xing, Jia, Wu and Kuang. This is an open-access article distributed under the terms of the Creative Commons Attribution License (CC BY). The use, distribution or reproduction in other forums is permitted, provided the original author(s) and the copyright owner(s) are credited and that the original publication in this journal is cited, in accordance with accepted academic practice. No use, distribution or reproduction is permitted which does not comply with these terms. 